\title{
THE EFFECT OF COVID-19 PANDEMIC ON THE EMOTIONS OF NURSES IN ISRAEL
}

\author{
Orly Zelevich ${ }^{1}$, Gadi Navon ${ }^{2}$, Halit Kantor ${ }^{3}$, \& Shulamith Kreitler ${ }^{4}$ \\ ${ }^{I}$ Department of Public Health, Ariel University (Israel) \\ ${ }^{2}$ Department of Social Sciences, The Open University (Israel) \\ ${ }^{3}$ Department of Communication studies, Tel-Aviv University (Israel) \\ ${ }^{4}$ School of Psychological Sciences, Tel-Aviv University (Israel)
}

\begin{abstract}
The Covid-19 pandemic, which originally spread in China in late 2019 and then affected the entire world including Israel, has thrown into the battle numerous medical teams, including physicians, nurses and other paraedical teams, both in hospitals and in the community. The medical personnel embarked on a variety of new tasks and challenges, which required them to manifest extraordinary strength. Healthcare providers and caregivers are one of the vital resources in each and every country. Their health and safety are important and crucial parameters not only for the continuous and safe care of patients, but also for controlling the outbreak of epidemics. Working in the medical field is known to bear implications for the mental health of healthcare providers and anxiety, depression, insomnia and stress are not a rare occurrence (S. Liu et al., 2020). Therefore, there is a need to consider the well-being of medical staff and to provide support where needed.
\end{abstract}

Keywords: Covid-19, nurses, emotions, fear, indispensability.

\section{Introduction}

Many studies have researched and are still researching the immense impact of the Covid-19 pandemic on our lives. Studies conducted among the general population, regardless of occupation, examined the psychological impact of the spread of the virus on the mental health of the general population. In Israel, too, the issue has been studied among the general population and a direct link has been found between fear of the virus and demographic variables such as gender, socio-demographic status, the existence of a chronic illness and having a close relative who has been severely affected by the virus (Tzur Bitan et al., 2020). On the one hand, nursing staff have the professional knowledge and mental strength to deal with critical health issues and, therefore, may fear the virus less when compared to the general public. On the other hand, nursing staff are exposed daily to many patients, some of whom may be carriers of the virus, which puts staff and their families at increased risk of infection, and therefore may result in increased negative impact on their mental health (Lai et al., 2020; Chidiebere et al., 2020,; El-Hage et al., 2020).

Nurses have been and continue to be at the forefront of this epidemic, and nursing personnel provide a wide range of essential services to patients, families and the community al large. The irony of the Corona plague now occurring during the International Nurses Year has not affected the teams, who have risen fearlessly and whose contribution to global health has never been clearer (Treston, 2020). During the Covid-19 pandemic, there has been a higher incidence of anxiety and stress among the medical staff. Medical institutions need to place emphasis on strengthening the psychological skills of medical personnel. Special attention should be paid to the mental health of nurses (Huang, Han, Luo, Ren, 2020 \& Zhou).

\section{Objectives}

The goal of the study was to investigate the psychological and emotional impact of the Covid-19 pandemic on nursing staff in Israel, with emphasis placed on feelings of fear.

The main research question, according to which the research hypotheses have been constructed, was whether there would be differences between nurses working in the community and nurses working in 
hospitals in terms of positive and negative emotions and the feeling of fear of Covid-19. The study takes into account the demographic variables and sense of indispensability among nurses.

Accordingly, there were four hypotheses.

1. High seniority of nursing staff in the community will be in a positive correlation with positive factors such as affection, joy and satisfaction and in a negative correlation with fear of Covid-19. The higher the seniority the less fear there will be.

2. There will be differences between nurses who work in hospitals and nurses who work in the community in terms of negative and positive emotions. However, it is hypothesized that both research groups will manifest an increase in feelings of indispensability and positive feelings of curiosity during the Corona period.

3. There will be no significant differences in the Corona Fear Questionnaire between the two groups.

4. There will be differences between nurses working in hospitals and nurses working in the community in terms of being indispensable at work, with the community nurses gaining a lower score on the feeling of "being seen" by the workplace.

\section{Design}

The study is a two-group descriptive quantitative study.

\section{Method}

\subsection{Method: Participants}

The target population of the study was nurses working in hospitals and in the community with similar age and educational characteristics. The sample included 203 participants, of whom 86 were hospital nurses and 117 were nurses working in the community setting.

\subsection{Method: Research tools}

Four research tools were used in the research: Demographic questionnaire, indispensability questionnaire, POMS (profile of mood states) questionnaire, and the Fear of COVID-19 Scale questionnaire, which was translated from English to Hebrew and back before submission to participants.

\subsection{Method: Research procedure}

After receiving the approval of the Ethics Committee, the questionnaires were made available to hospital and community nurses online, using the snowball method. Participants were asked to complete the questionnaire after they had received an explanation of the purpose of the study and given informed consent to participate in the research anonymously. The data obtained was then submitted for statistical analysis.

\section{Results}

The study compared negative and positive emotions and the feeling of fear of Covid-19 with reference to demographic variables such as seniority, education, type of contract (full-time or part-time), sense of indispensability and job satisfaction. A factor analysis showed that the Fear of Covid-19 scale included two factors. One included four items referring to physical syumptoms (Mean=40.84, SD=11.76, eigenvalue $=3.58$ ), while the second included 3 items referrring o fears of eh visrus (Mean=47.450, $\mathrm{SD}=17.26$, eignvalue $=1.017$ ). There were no significant differences in these two factors between nurses in the hospitals and in the community. Significant and inverse relationships have been found between negative emotions and age; as age increases so does the level of negative emotions during routine as well as during the Covid-19 pandemic (with this relation being stronger during the pandemic). Similarly, a weak inverse relationship was found between age and fear of Covid-19- as age increases fear of Covid-19 decreases. In both groups of nurses there was a significant decline in positive emotions during the pandenic period $(\mathrm{F}(1,201)=93.24, \mathrm{p}=0.000$.). Comparing settings, a significant difference has been found in the level of negative emotions, with nurses working in hospitals having more negative emotions compared to nurses working in the community, whereas positive emotions were more common among nurses working in the community compared to nurses working in hospitals. During the pandemic there was an increase in negtive emotosn, in particualr sadnes and difficulty to concentrate. 
Table 1. Regression analysis of variables contributing to Satisfaction with one's work at present.

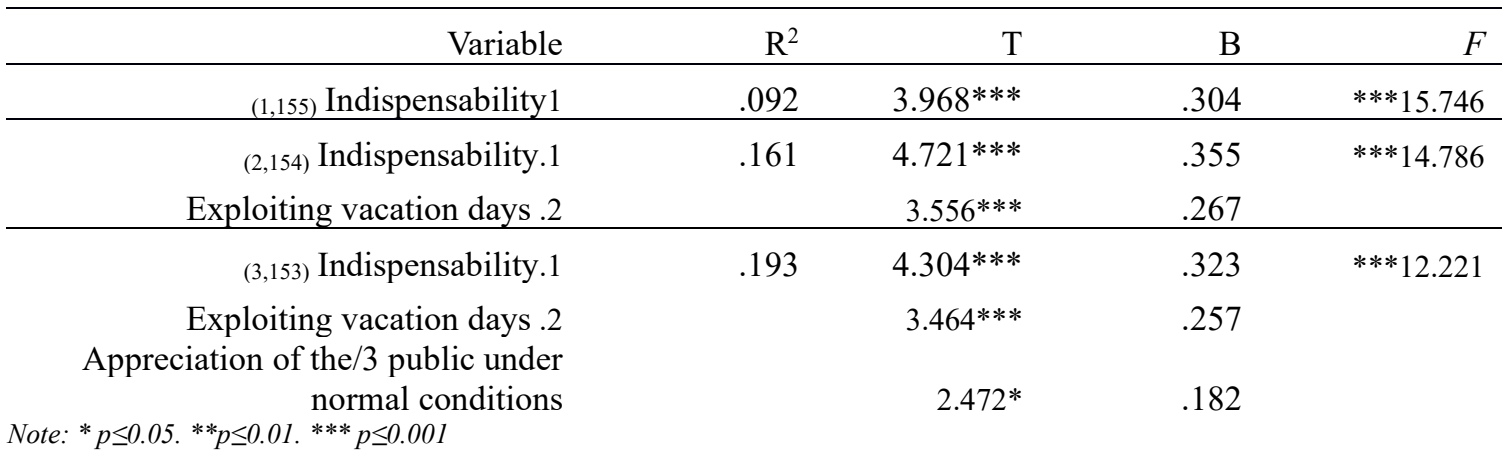

As for sense of indispensability and satisfaction, nurses felt that their needs during the Covid-19 period have not gained sufficient attention from the employer compared to the routine period, and this difference was also found to be significant both in the hospital and in the community settings. Table 1 shows that in addition, nurses in both settings felt a significant increase in public appreciation for their work during the Covid-19 period compared to routine. Figure 1 shows that there was a difference in the sense of indispensability between nurses in eh community and hospital nurse. The former felt more indispensable.

Figure 1. Comparison between community nurses and hospital nurses in indispensability.

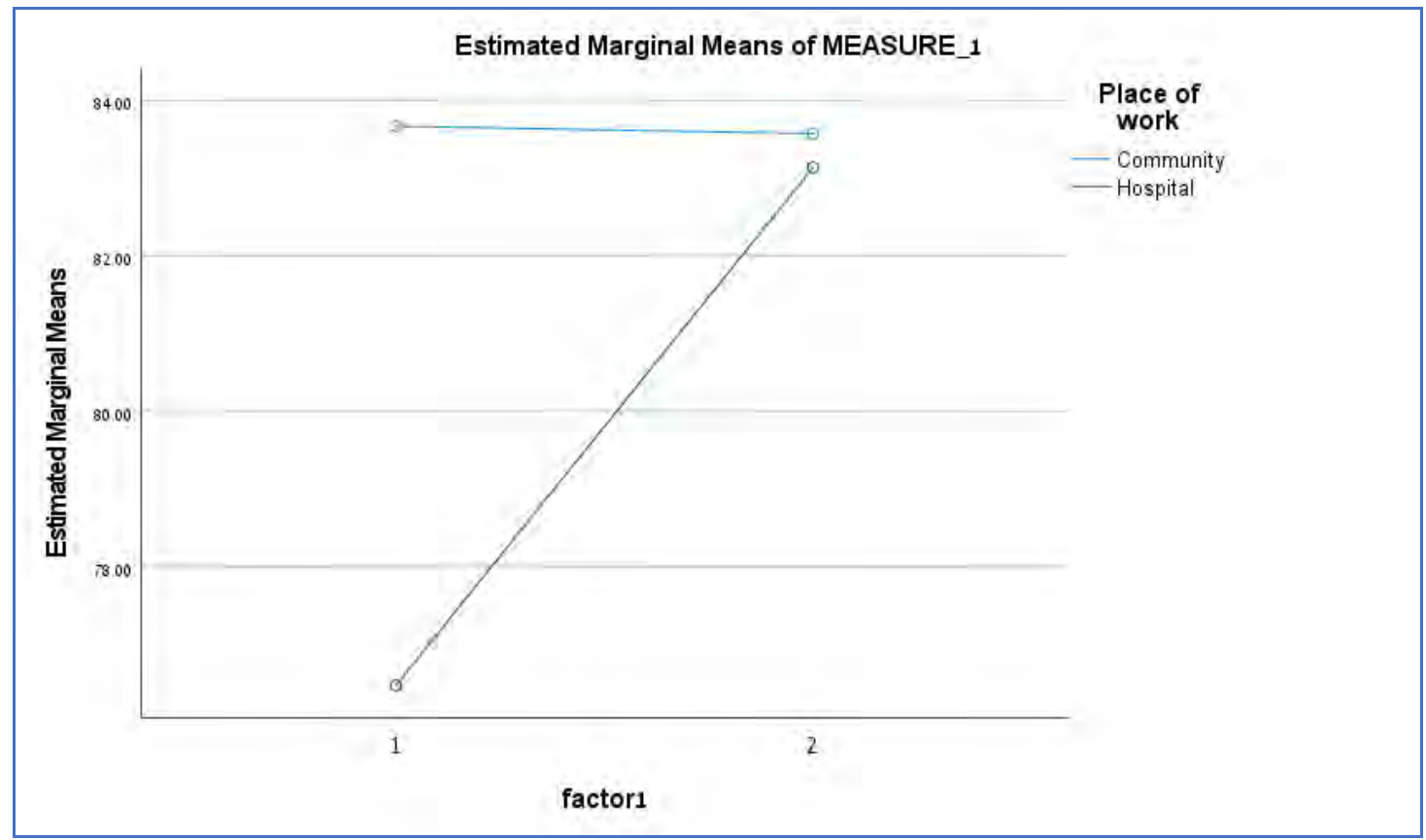

A comparison was made between the period before the pandemic and during the pandemic in the sense of indispensability between nurses differing in seniority. The difference was significant $\mathrm{F}(2,199)=5.879, \mathrm{p}=.003$ ). In those with longer seniority (over 11 years) the mean gap between before and during the pandemic was small (Mean $=.845, \mathrm{SD}=10.49$ ) while in those with shorter seniority (less than 5 years), the gap was larger (Men=7.48, $\mathrm{SD}=13.49)$. Figure 2 shows clearly this effect of seniority. 
Figure 2. The effect of seniority on the feeling of indispensability of nurses.

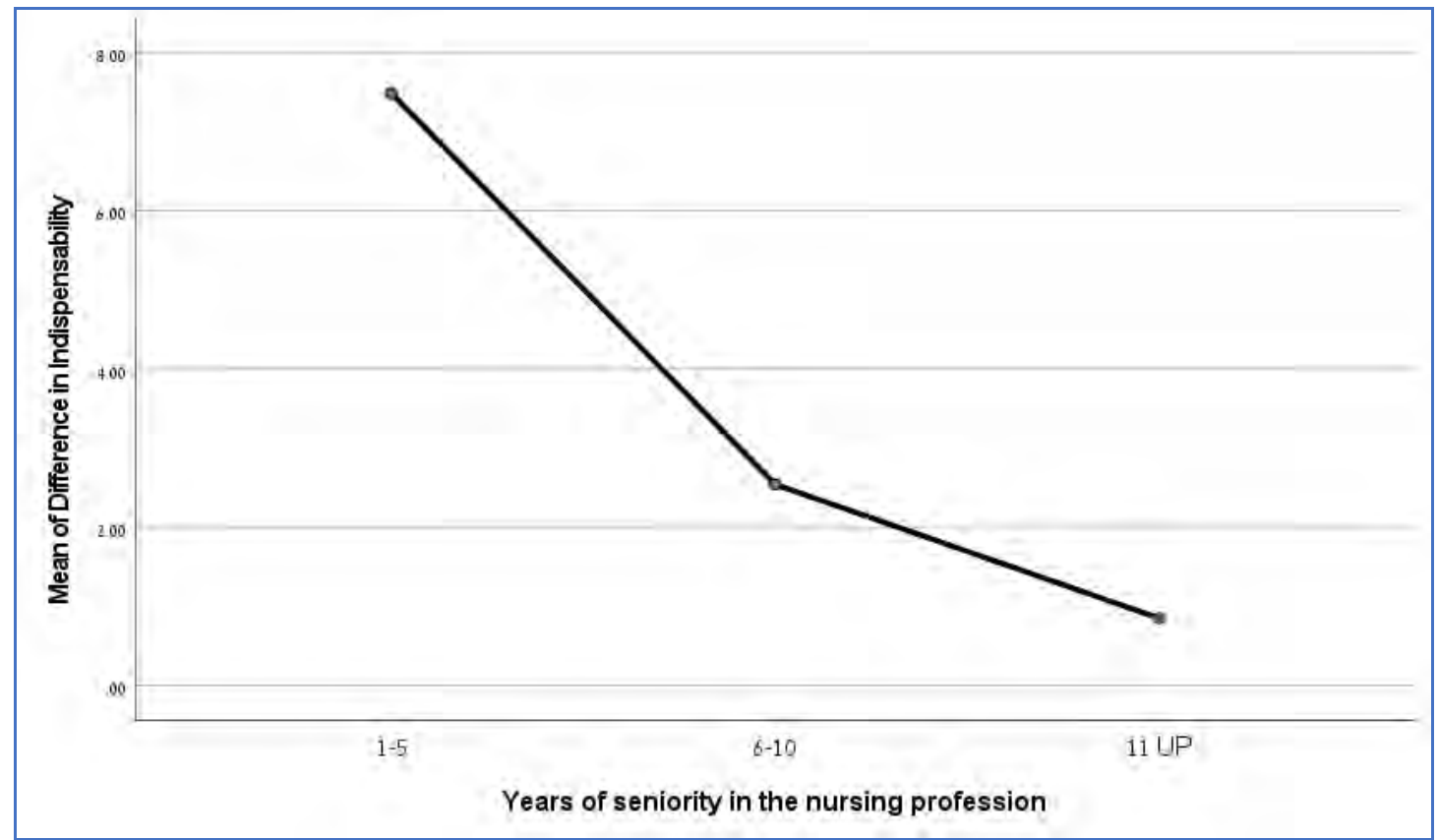

Regarding gender, no significant differences in emotional reaction have been found between men and women (Lasalvia et al., 2020).

\section{Discussion and conclusions}

Nurses working in the community experience more positive feelings compared to nurses working in hospitals, who in turn experience significantly more negative emotions in routine and during the Covid-19 pandemic. The findings of the study suggest that community nurses have more joy and enjoyment at work, while nurses working in hospitals have a greater sense of commitment and indispensability. Thus, there are gaps in feelings and emotions between the two settings and, accordingly, different measures are required (Pollock et al., 2020; Tracy et al., 2020): As for the hospital setting, intervention programs should be considered to reduce negative feelings among nurses and to intensify positive emotions; In the community setting, an effort should be made to raise the sense of indispensability that exists among hospital nurses. The current study entails practical implications and its significance lies in raising awareness to the needs of nurses - in both settings - in urgent and routine times.

\section{References}

Chidiebere Okechukwu E, Tibaldi L, La Torre G. The impact of COVID-19 pandemic on mental health of Nurses. Clin Ter. 2020 Sep-Oct;171(5):e399-e400. doi: 10.7417/CT.2020.2247. PMID: 32901781.

El-Hage, W., Hingray, C., Lemogne, C., Yrondi, A., Brunault, P., Bienvenu, T., ... \& Aouizerate, B. (2020). Health professionals facing the coronavirus disease 2019 (COVID-19) pandemic: What are the mental health risks?. Encephale, S73-S80.

Huang, J. Z., Han, M. F., Luo, T. D., Ren, A. K., \& Zhou, X. P. (2020). Mental health survey of 230 medical staff in a tertiary infectious disease hospital for COVID-19. Zhonghua lao dong wei sheng zhi ye bing za zhi= Zhonghua laodong weisheng zhiyebing zazhi= Chinese journal of industrial hygiene and occupational diseases, 38, E001-E001.

Lasalvia A, Bonetto C, Porru S, Carta A, Tardivo S, Bovo C, Ruggeri M, Amaddeo F. Psychological impact of COVID-19 pandemic on healthcare workers in a highly burdened area of north-east Italy. Epidemiol Psychiatr Sci. 2020 Dec 17;30:e1. doi: 10.1017/S2045796020001158. PMID: 33331255 ; PMCID: PMC7804082. 
Liu, Q., Luo, D., Haase, E.J. et al. (2020) The experiences of health-care providers during the COVID-19 crisis in China: a qualitative study. The Lancet, volume 8, issue 6, e790-e798, JUNE 01. Open access:April 29, DOI:https://doi.org/10.1016/S2214-109X(20)30204-7.

Pollock, A., Campbell, P., Cheyne, J., Cowie, J., Davis, B., McCallum, J., ... \& Maxwell, M. (2020). Interventions to support the resilience and mental health of frontline health and social care professionals during and after a disease outbreak, epidemic or pandemic: a mixed methods systematic review. Cochrane Database of Systematic Reviews, (11).

Tracy, D. K., Tarn, M., Eldridge, R., Cooke, J., Calder, J. D., \& Greenberg, N. (2020). What should be done to support the mental health of healthcare staff treating COVID-19 patients?. The British Journal of Psychiatry, 217(4), 537-539.

Treston, C. (2020). COVID-19 in the Year of the Nurse. J Assoc Nurses AIDS Care. Apr 21. doi: 10.1097/JNC.0000000000000173.

Tzur Bitan, D. T., Grossman-Giron, A., Bloch, Y., Mayer, Y., Shiffman, N., \& Mendlovic, S. (2020). Fear of COVID-19 scale: Psychometric characteristics, reliability and validity in the Israeli population. Psychiatry Research, 289, 113100. 